\title{
Bullying: políticas de atención y prevención en Costa Rica
}

\author{
Bullying: attention and prevention policies in Costa Rica
}

\author{
María Martha Durán ${ }^{1}$ \\ Universidad Estatal a Distancia, Costa Rica
}

\begin{abstract}
Resumen. Hay evidencia de la existencia de bullying en las instituciones educativas costarricenses, aunque no hay referencia a investigaciones epidemiológicas o psicométricas específicas; por ello que no hay claridad sobre su dimensión real ni los factores asociados que orienten las políticas públicas propuestas en los últimos años. Además, existen problemas conceptuales respecto al término de bullying y en relación con el diseño, ejecución y control de programas en este tema. Las implicaciones son variadas e importantes, por ejemplo, la desatención a las situaciones concretas y/o el manejo inapropiado de estas cuando se presentan, lo cual propicia la revictimización por un lado y la impunidad por el otro; además, la generación de un ambiente hostil que incide en el proceso de enseñanza-aprendizaje e inseguridad y desconfianza en el sistema educativo. Ante esto se requieren intervenciones urgentes y concertadas desde diferentes niveles y perspectivas para la revisión y replanteamiento de todas estas falencias; además de que se reflejen en políticas públicas acordes a nuestra realidad, fundamentadas en investigación nacional y regional, con estrategias que puedan ser evaluadas con criterios objetivos basados en la prevención, pero con procedimientos claros para la atención y mitigación del bullying.
\end{abstract}

Palabras clave: matoneo, Costa Rica, situación actual, políticas públicas, propuestas.

\begin{abstract}
Although there is evidence of bullying in Costa Rican educational institutions, there is no reference to specific epidemiological or psychometric research; therefore, there is no clarity about its real dimension or associated factors to guide public policies that have been proposed in recent years. Furthermore, there are also conceptual issues regarding the definition of bullying and in relation to the design, implementation and program monitoring on this subject. Implications are varied and important, for example, neglect to specific situations and / or improper handling when these arise, which favors re-victimization on one hand and impunity on the other; the creation of a hostile environment that affects the teaching-learning process and the insecurity and distrust in the education system. Urgent and concerted actions are required from different levels and perspectives for the revision and reformulation of the aforementioned shortcomings. Those actions are to be reflected in public policies consistent with our reality, based on national and regional research, with strategies that can be assessed with objective criteria based on prevention, but with clear procedures for the attention and mitigation of bullying.
\end{abstract}

Keywords: bullying, Costa Rica, current situation, public policies, proposals

${ }^{1}$ María Martha Durán, Psicóloga con especialidad en Trabajo y Organizaciones, doctora en Educación. Directora de la Revista Posgrado y Sociedad del Sistema de Estudios de Posgrado de la Universidad Estatal a Distancia. . E-mail: mduranr@uned.ac.cr 


\section{María Martha Durán}

\section{Introducción}

Los fenómenos de violencia en los centros educativos no son un tema nuevo. El bullying o maltrato escolar entre pares² (Durán, 2015) es una de las formas de violencia escolar más estudiadas desde los años 70 a partir de los estudios de Heinemann, Olweus y Pikas (Salmivalli, Lagerpetz, Björkqvist, Österman \& Kaukiainen, 1996; Roland, 2010) por su condición de daño, su frecuencia, intencionalidad e impacto.

En los años 90, se empezó a dar mayor visibilidad al problema debido al aumento en la proporción de estudiantes violentos, la intensidad y forma de sus actos de violencia hacia compañeros y profesores en el propio centro educativo (Hanewinkel y Eichler, 1999, citados por Fuchs, 2009). Además, el estudiantado se incorpora cada vez más temprano, lo cual prolonga el período de institucionalización y con ello la exposición a situaciones de riesgo y de bullying (Magendzo y Toledo, 2007). A esto se suma el impulso que generan las nuevas tecnologías en cuanto a que pueden exponer a los niños, niñas y adolescentes con demasiada frecuencia a todo tipo de violencia, permitiendo además que estos hechos se conozcan de manera más fácil y expedita. Por ejemplo, a partir de las fotografías y filmaciones captadas desde teléfonos celulares o cámaras digitales por los propios estudiantes, y que se publican en internet de diferentes formas (Grillo y Esquivel, 2011).

La notoriedad pública del fenómeno vinculada a la exposición mediática ha impulsado la investigación creciente y propiciado diferentes niveles de presión por acciones concretas que lo minimicen y evite apariciones posteriores. Esto

${ }^{2}$ Por ser un término en inglés, no hay una palabra en español que denote de forma exacta el significado del término bullying. Sin embargo, y siguiendo los resultados del estudio de Sánchez y Ortega (2010), se asume el término "maltrato" como su equivalente en este idioma y se referirá a "Maltrato Escolar entre Pares" (MEeP) para delimitar el ámbito de interés de este artículo. ha permitido el reconocimiento por parte de un sector importante de la comunidad y ha posibilitado la generación de acciones para intentar transformarlo, paliarlo o eliminarlo al modificar las prácticas desde las cuales el bullying emerge. Sin embargo, también se ha generado mayor confusión al asumir violencia, agresión y conflicto como sinónimos.

La primera de las limitaciones fundamentales para el abordaje de este tema es, precisamente, la confusión semántica y lingüística. La falta de claridad conceptual y su delimitación frente a otros términos similares ha propiciado el uso de gran variedad de nombres que refieren a comportamientos diferentes, pero similares, configurando así un "crisol semántico" (Sánchez y Ortega, 2010; Taki, Slee, Hymel, Pepler, Sim y Swearer, 2008). Esto incide en su concepción y metodología para la investigación.

La segunda es la "cultura del silencio" (Benites, Carozzo, Horna, Palomino, Salgado, Uribe y Zapata, 2012; Durán, 2014; Magendzo y Toledo, 2007; Piñuel y Oñate, 2007) la cual, basada en mitos y estereotipos, ha ayudado a la perpetuación del bullying en tanto que dificulta su reconocimiento y limita las denuncias por este motivo, facilitando así que se mantenga oculto. Por último, el restringir la mirada a la díada matón-víctima deja de lado otros componentes fundamentales de la situación y sus roles (Salmivalli et al., 1996).

Para este artículo, y según se ha planteado en otros escritos (Durán 2014, 2015), se entiende por bullying a un problema multifactorial y procesual de violencia interpersonal escolar entre pares o iguales (estudiantes) que conviven cotidianamente en el ámbito educativo. Se ejerce de forma proactiva y prolongada; además su objetivo es causar daño mediante agresiones sistemáticas, intencionales, reiterativas y unidireccio- 
nales que realizan una o varias personas contra otra que se encuentra indefensa, vulnerable.

Aun cuando suelen tener un mismo estatus, quien agrede toma el control de la interacción a partir de la reproducción de una situación de desequilibrio de poder (real o percibido, de poder, fuerza o número). Esta, al ser reiterada e inexplicable (no hay una causa concreta y clara que la víctima pueda identificar) genera miedo o temor de la víctima, haciéndola impotente para salir de la situación por sí sola. ${ }^{3}$

La intención de daño se manifiesta a través de acciones directas o indirectas en cinco ámbitos: físico, verbal, social o relacional, psicológico y sexual; también puede presentarse una combinación de estas formas. El grado de intensidad varía $\mathrm{y}$, en tanto fenómeno procesual, tiende a incrementarse pasando de acciones ligeras a moderadas y graves; aunque todas son el igualmente dañinas y corrosivas para quien las sufre.

En cualquiera de sus manifestaciones, el bullying atenta contra la dignidad y los derechos fundamentales de quien sufre del maltrato, provocando en las víctimas efectos claramente negativos en los diferentes ámbitos de su vida (personal, familiar, social, académico). Además, daña el buen desarrollo y el equilibrio emocional, intelectual y social de todos los implicados (incluyendo el clima negativo en el aula que genera).

El abordaje asumido se enfoca desde la prevención, considerando a la Convivencia Escolar como el eje desde el cual se debe actuar. Esto debido a sus dos funciones principales: en primer lugar formativa, en cuanto a la enseñanza de la interacción basada en el respeto a los derechos

${ }^{3}$ En tanto proceso, es la repetición de la agresión de los mismos matones sobre la misma persona, más que la intensidad del hecho, lo que genera impotencia en la víctima para salir de esa situación por sí misma. La duración del maltrato provoca desgaste emocional e indefensión en la víctima (miedo, ansiedad y/o tristeza), que progresivamente van a ir minando su resistencia. humanos; en segundo lugar, desde una perspectiva de cultura de paz, propicia una ciudadanía responsable (Chaux, 2012).

\section{Bullying en Costa Rica}

La violencia escolar y el bullying, como su manifestación más importante, están presentes en las instituciones educativas costarricenses y afectan seriamente la convivencia escolar. En la tabla 1 se presentan los porcentajes reportados en las investigaciones analizadas respecto a la proporción entre actores.

Los estudios de Cabezas (2007) y Cabezas y Monge (2007) con estudiantes de colegio de la Gran Área Metropolitana costarricense miden las conductas agresivas y brindan datos sobre las personas a quienes se victimiza. Algunos de los resultados obtenidos evidencian que la mayoría de los agresores son hombres (21\%) y utilizaron más la fuerza física; $9 \%$ son mujeres y acosaban a sus iguales de forma solapada. El tipo de violencia que con mayor frecuencia se presentó en esos grupos de estudiantes fue la verbal (a través de los insultos), seguida de empujones y los golpes.

Es importante resaltar que, en la mitad de las ocasiones, las personas agredieron a otro (s) no tuvieron ninguna consecuencia por sus actos. Esta situación muy riesgosa en términos de tolerancia e impunidad pues, al no aplicarse medidas correctivas que impidan esta acción, se socava la autoestima y rendimiento de quienes las padecen. Respecto a las víctimas en secundaria, se obtuvo que del total de participantes 33\% se identifica como tal $-17 \%$ varones y $15 \%$ mujeres-, los cuales viven las agresiones en presencia de otros, quienes permanecen al margen de la situación.

En 2012 Cabezas y Monge (2013) realizaron una nueva investigación tomando los datos de 


\section{María Martha Durán}

Tabla 1.

Comparación de datos de diferentes estudios sobre violencia escolar y bullying en actores de secundaria en Costa Rica.

\begin{tabular}{lcccc}
\hline & $\begin{array}{c}\text { Cabezas, 2007; } \\
\text { Cabezas y } \\
\text { Monge, 2007. } \\
\text { GAM*, CR } \\
\mathrm{N}=371\end{array}$ & $\begin{array}{c}\text { Cabezas y } \\
\text { Monge, 2013 } \\
\text { GAM,CR } \\
\text { N=1.155 }\end{array}$ & $\begin{array}{c}\text { Lentini et al., } \\
2012, \mathrm{CR} \\
\mathrm{N}=801\end{array}$ & $\begin{array}{c}\text { Durán, 2015 } \\
\text { Cartago, CR }\end{array}$ \\
\hline Agresores & $17 \%$ & $20 \%$ & $9 \%$ & \\
Víctimas & $33 \%$ & $41 \%$ & $39 \%$ & $28 \%$ \\
Testigos & - & $* *$ & $71 \%$ & $61 \%$
\end{tabular}

Nota. Durán, 2015; con base en datos de Cabezas (2007), Cabezas y Monge (2007), Cabezas y Monge (2013),

Lentini y colaboradoras (2012) y datos propios.

* Gran área metropolitana

**Se hace referencia a testigos, pero no se especifican porcentajes.

investigaciones previas y los compararon con los datos obtenidos en un nuevo estudio realizado con 1155 estudiantes con edades entre 8 y 15 años en instituciones de la Gran Área Metropolitana. Se encontraron diferencias significativas en los resultados comparativos los cuales permiten afirmar que el matonismo es una realidad que va en aumento en el país.

En el estudio de Lentini, García y Borbón (2013) sobre violencia escolar realizado en 2012 para el IV Informe Estado de la Educación, se confirma que la violencia en sus diversas modalidades es parte de la convivencia diaria en los centros educativos (39\% de los estudiantes ha sufrido algún acto violento y $9 \%$ son víctimas frecuentes). Además, en mayor o menor medida, tanto docentes como estudiantes participan de ella. Los malos tratos verbales -burlas y humillaciones constantes- son más frecuentes que los físicos.

Los hombres son más propensos a ser víctimas de violencia que las mujeres. Sus reacciones principales son no hacer nada o hablar con alguien al respecto y es mínima la respuesta de denuncia. Se refiere que es clara la tendencia a considerar solamente las conductas de violencia física como indicador de violencia escolar. La violencia física como indicador, el tamaño del colegio -en cuanto a la cantidad de estudiantes y la relación con el espacio físico- y la ausencia de apoyo docente y de los directores, aumentan el riesgo de aparición de situaciones de violencia escolar, según las autoras. ${ }^{4}$

Durán (2015) presenta resultados de investigación con estudiantes de dos colegios diurnos de la provincia de Cartago durante el período lectivo 2013 los cuales confirman la presencia de bullying en las instituciones educativas costarricenses. Respecto a formas de interacción y situaciones de agresión entre pares se obtuvo que seis de cada diez estudiantes de octavo año de esos colegios han presenciado alguna acción

\footnotetext{
${ }^{4}$ Aunque las variables incluidas en el análisis no determinan la probabilidad de victimización ciertas características de las personas o del centro educativo sí muestran un grado de asociación. Por ejemplo, el tamaño del colegio (entre 350 y 500 estudiantes) aumenta ese riesgo, mientras que cursar el sétimo año y contar con apoyo docente tiende a disminuirlo. Además, el estudio permite descartar algunos factores, de modo que ser víctima no depende del sexo, el estrato socioeconómico, el tipo de institución o el lugar donde esta se ubica.
} 
de maltrato entre compañeros o compañeras en el último mes, mientras que tres lo han sufrido como víctima y uno se identifica como agresor (esto indica que un mismo agresor puede estar victimizando a varios estudiantes).

Estas acciones están matizadas por género: los varones son con mayor frecuencia agresores y también son con mayor frecuencia víctimas. En cambio, las mujeres tienden a ser más sensibles como espectadoras, captan más conductas bullying. Las manifestaciones principales identificadas son verbales (burlas, reírse ante una equivocación, criticar y decir apodos que ofenden o ridiculizan), las cuales son el medio para concretar otras formas más sutiles como la violencia psicológica y social. Se identifica al equipo docente, la familia y aspectos personales como fundamentales en la dinámica del bullying.

Por último el Ministerio de Educación Pública cuenta con datos estadísticos sobre violencia escolar que reflejan un importante incremento en las denuncias en los últimos años (Solís, 2013; Contraloría de Derechos Estudiantiles, MEP, 2014, 2015). De las denuncias presentadas en 2012 por violencia escolar entre estudiantes, 57 fueron por matonismo; en el 2013 de un total de 408 reportes, 210 fueron por el mismo motivo (4 de estas por cyberbullying). En el año 2014 se presentaron 409 denuncias por situaciones de violencia en centros educativos y 229 correspondían a matonismo (incluyendo 14 por cyberbullying). En 2015 se reportaron 240 casos de acoso escolar y 8 por cyberbullying.

\section{Respuesta estatal ante la situación del bullying}

Desde la perspectiva legal, Costa Rica cuenta con un importante marco normativo referente a la Niñez y Adolescencia en el ámbito educativo, el cual parte de la Convención Internacional sobre los Derechos del Niño. Además incluye la
Constitución Política de Costa Rica, la Ley Fundamental de Educación, el Código de la Niñez y la Adolescencia, la Ley de Justicia Penal Juvenil, la Ley contra el Hostigamiento Sexual en el Empleo y la Docencia y su Reglamento en el sector educativo (MEP, 2012), la Ley sobre Resolución Alternativa de Conflictos y el Reglamento de Evaluación de los Aprendizajes (MEP, 2009), entre otros. ${ }^{5}$

Acciones puntuales están generando cambios en términos de desarrollo de normativa específica:

i) Pronunciamiento de la Sala Constitucional (Sentencia 2013-10711), por un voto de mayoría. Los niños, niñas y jóvenes que denuncien matonismo en sus escuelas o colegios tendrán derecho a mantenerse en el anonimato "toda vez que esta es la opción que más beneficia al menor víctima de bullying, en la medida en que significa el resguardo preventivo y necesario de su integridad y su dignidad contra eventuales represalias de tipo físico, psicológico o de cualquier naturaleza por parte del agresor denunciado" (Ross, 16 de agosto, 2013)

iii) En julio 2014, se conoció la primera sentencia contra el Estado por omisión de los docentes para atender el bullying en el aula, ante la agresión verbal y física que sufrió una estudiante de sétimo año, en el 2012 (Sentencia 59-2014-IV). Los jueces condenaron al Estado a pagar todos los gastos por atención psicológica de la menor y costos de traslado del colegio. Por su parte, los estudiantes que agredieron física, verbal y sexualmente a la estudiante enfrentaron un proceso penal juvenil. La joven víctima fue trasladada en 2013 a otro centro educativo (Barrantes, 09 de agosto, 2014; Durán \& Vargas, 2015).

iii) La Sentencia 2015-005234 de mayo de 2015 sobre el derecho a la Educación y la protección

${ }^{5}$ Se suman dos iniciativas presentadas ante la Asamblea Legislativa referidas a proyectos de Ley: la primera es la "Ley especial para la Protección de los derechos de la niñez y la adolescencia frente a la violencia y el delito en el ámbito de las Tecnologías de la Información y la Comunicación y Reformas al Código Penal" (expediente 18230). La segunda fue presentada en noviembre 2014 (expediente \#19399) “Ley para prevenir, erradicar y sancionar el Acoso Estudiantil” (Ley Bullying). 
de un estudiante que es víctima de agresiones por parte de otro, obliga a tomar las medidas necesarias de protección, atención de la violencia y rehabilitación de estudiantes víctimas y victimarios de ese tipo de situaciones en el centro educativo. Con ello se garantiza el proceso educativo integral y adecuado para el amparado y los demás estudiantes.

Por otra parte, desde el Ministerio de Educación Pública (MEP) las acciones ante la violencia escolar parten en 2009 y se reflejan en el Reglamento de Evaluación de los Aprendizajes (REA, 2009). En este, se asume que es un derecho del estudiantado "...recibir de los docentes, funcionarios y compañeros, un trato basado en el respeto a su integridad física, emocional y moral así como a su intimidad y a sus bienes" (Art. 19.c) y deben "...practicar con esmero las normas de consideración y respeto en sus relaciones con los compañeros, con los profesores, personal, autoridades de la institución y en general, con todas las personas". También, en este documento, se definen los tipos de faltas a sancionar, por ejemplo: las faltas graves (art. 76) se refieren a acciones y actitudes graves de indisciplina contra "los alumnos, incluyendo frases o hechos irrespetuosos dichos o cometidos en otros estudiantes". Entre las faltas muy graves se incluyen aquellas acciones que inciten a los compañeros a participar en acciones que perjudican la salud, seguridad individual o colectiva y cualquier tipo de acción discriminatoria (por raza, credo, género, discapacidad o cualquier otra contraria a la dignidad humana). Por último las faltas gravísimas se relacionan con la reiteración de "la destrucción deliberada de bienes pertenecientes a $[\ldots]$ los demás miembros de la comunidad educativa, ya sea que la acción sea individual o en grupo", además de la agresión física contra cualquier miembro de la comunidad educativa. Es evidente el énfasis en aspectos referidos a violencia física y patrimonial.
En 2010, el Gobierno costarricense empiezó a gestar acciones paliativas frente al bullying a partir de las estadísticas sobre casos de violencia entre estudiantes en el período 2003-2009 del MEP (III Informe Estado de la Educación, 2011) y el seguimiento posterior. Así, en julio de 2010 se dictó la Circular DM-005-07-2010 sobre las Acciones Institucionales para la Convivencia en los Centros Educativos, sustentado en el artículo 2 de la Ley Fundamental de Educación y considerando que los centros educativos constituyen escenarios de formación ciudadana para el fortalecimiento de las buenas prácticas de convivencia a partir del desarrollo de procesos de aprendizaje y habilidades interpersonales.

Se insta entonces a reafirmar los valores éticos, estéticos y ciudadanos como base de la convivencia escolar, tal y como lo postula la Política Educativa promulgada por el Consejo Superior de Educación, con la elaboración de reglamentos internos en cada institución. Surgen programas de atención y prevención como "El cole en nuestras manos", programa participativo que se está desarrollando en 63 colegios de todo el país considerados de atención prioritaria. También, está el Proyecto "Ética Estética y Ciudadanía", el cual pretende desarrollar en el estudiantado la sensibilidad, destrezas y habilidades necesarias para saber vivir y convivir.

En 2011, nació el Programa Nacional de Convivencia en Centros Educativos (Programa Convivir) que en 2012 dio a conocer el "Protocolo integrado para la atención de situaciones de violencia en los centros educativos de secundaria". Este, a su vez, está constituido por cuatro protocolos específicos: contra la violencia física, psicológica y sexual; contra el acoso, matonismo o bullying y el ciberbullying; contra el uso y tráfico de drogas y, por último, contra la portación y uso de armas. Estos protocolos están acompañados de sesiones de capacitación. Ese mismo año 
el Ministerio de Educación Pública y el Patronato Nacional de la Infancia (PANI) impulsaron la campaña de Visión Mundial y Cartoon Network "Basta de bullying, no te quedes callado" (http:// www.bastadebullying.com).

En setiembre de 2013 se realizó en Costa Rica el Foro Iberoamericano para prevenir situaciones de Bullying, coordinado por el MEP y con el apoyo del Banco Interamericano de Desarrollo, la Organización de las Naciones Unidas para la Educación, la Ciencia y la Cultura (UNESCO) y el Fondo de las Naciones Unidas para la Infancia (UNICEF). En ese espacio para el intercambio de experiencias sobre la prevención y atención del bullying en la comunidad educativa, participaron docentes costarricenses, representantes de los Ministerios de Educación de Honduras, El Salvador, Guatemala, Panamá y Uruguay y se discutieron las experiencias de Costa Rica, Argentina, Chile, Colombia y México (el material esta accesible en http://www.mep.go.cr/foro-bullying).

Durante el 2014, se desarrolló una serie de videos informativos sobre el tema, dirigidos a estudiantes y a adultos ("Sin Etiquetas", "No al Bullying", "Me gusta el color de tu piel") y se habilitó un correo electrónico para denuncias (derechosestudiantiles@mep.go.cr). En 2015 se inició un programa de formación y actualización virtual y bimodal dirigido a docentes, en el cual se incluye un curso específico, "Convivencia". Este, busca fortalecer la convivencia escolar desde la perspectiva de derechos humanos y cultura de paz mediante la implementación de técnicas y estrategias vinculadas con el Programa Convivir. Además, en octubre de 2015 el Ministerio de Educación Pública presentó a la comunidad educativa una actualización del protocolo de prevención y atención en situaciones de bullying y el año 2016 fue declarado "Año de lucha contra el bullying".
El Patronato Nacional de la Infancia (PANI) es la instancia rectora en materia de Atención, Protección, Promoción, Prevención y Garantía de los derechos de los Niños, Niñas y Adolescentes en Costa Rica. Se fundamenta en el Código de la Niñez y la Adolescencia vigente desde 1998, que en el artículo 170 establece la creación del Consejo Nacional de Niñez y Adolescencia (CNNA) como una instancia de deliberación, concertación y coordinación entre el Poder Ejecutivo, las instituciones gubernamentales, instituciones autónomas y sociedad civil.

El objetivo del CNNA es la aplicación de políticas públicas y programas basados en el principio fundamental del respeto a los Derechos de la Niñez y Adolescencia. Está conformado por 22 entidades gubernamentales, no gubernamentales y de la sociedad civil y fue reactivado en 2014. En el año 2009 se decretó la implementación de los Subsistemas Locales de Protección a la Niñez y la Adolescencia, en los que se fomenta una estructura que articula lo nacional, lo local y lo regional.

El PANI brinda atención de consultas y denuncias directas y electrónicas en sus oficinas distribuidas en todo el país y mediante tres líneas telefónicas (emergencias, atención de consultas sobre los derechos de las personas menores de edad y la línea Adolescente Madre). Además, tiene una cuenta en redes sociales (https://www. facebook.com/PANICR) desde donde se presentan dos aplicaciones creadas en 2013: "Denuncias" y "Caceriadepedobear", microsite para reportar las páginas en Facebook que denigran la imagen de las personas menores de edad y actuar a través de la Fiscalía de Delitos Sexuales y ser reportadas ante la administración de Facebook para su eliminación. En redes sociales se difunde información básica sobre bullying; sin embargo, a pesar de su investidura, el PANI no ha desarrollado políticas específicas al respecto. 
El Viceministerio de Paz del Ministerio de Justicia y Paz cuenta con cuatro programas adscritos a la Dirección General para la Promoción de la Paz y la Convivencia Ciudadana (Digepaz). Se integra investigación y acción en cuatro áreas: Cultura de Paz, Prevención de Violencia Juvenil, Gestión Local, Observatorio de la Violencia. Ademas, se cuenta con un Plan Nacional de Prevención de la Violencia y Promoción de la Paz Social, una Dirección Nacional de Resolución Alterna de Conflictos (DINARAC), el Programa de Casas de Justicia y una Comisión Nacional para la Prevención de la Violencia y la Promoción de la Paz Social (CONAPAZ). No se identificaron acciones específicas en la temática bullying.

No obstante, se han desarrollado importantes trabajos de prevención e identificación de violencia escolar, aportando herramientas como el "Manual de procedimientos para la detección y denuncia de violencia y abuso contra personas menores de edad desde el centro educativo", elaborado por Ortiz, Solís y Umanzor (2004).

Asumiendo la violencia como un problema de salud pública (Organización Mundial de la Salud, 2004) que repercute en el ámbito educativo en donde se manifiesta de múltiples formas, se investigó sobre directrices desde el Ministerio de Salud. Si bien no hay una referencia concreta al bullying, la Ley General de la Persona Joven (2002) establece en el artículo 4-k el derecho a convivir en un ambiente sano y participar de las acciones que contribuyan a mejorar su calidad de vida. Además, los artículos 5 y 6 de señalan como deberes del Estado con esta población la atención de salud, trabajo y educación. Por otra parte, a pesar de que en este Ministerio se considera a la Salud Mental como un derecho, ni en el Plan de Acción del Plan Estratégico Nacional de Salud de las Personas Adolescente 2010-2018 (PENSPA) (2010) ni en la Política Nacional de Salud Mental 2012-2021 lanzada en octubre 2012, se contemplan temáticas referidas a Violencia Escolar en general o bullying en específico.

La Defensoría de los Habitantes de la República cuenta con una Dirección de Niñez y Adolescencia, la cual tiene por mandato institucional que velar que el funcionamiento del sector público garantice los derechos de los niños, niñas y adolescentes; además de vigilar las disposiciones del Código de la Niñez y la Adolescencia. Esta Dirección trabaja bajo cuatro ejes de acción. En primer lugar, recepción, procesamiento y resolución de consultas, denuncias y quejas, entendidas como solicitudes de intervención. En segundo lugar, promoción, capacitación y difusión de los derechos e intereses de la niñez y la adolescencia. En tercer lugar, seguimiento, revisión y reformulación de la legislación, normas y procedimientos institucionales en la materia. Por último, la promoción y acompañamiento de procesos sociales e institucionales dirigidos a la protección. A pesar de esto el aporte en materia de matoneo y cibermatoneo es baja, limitándose a generar información para la comunidad nacional a partir de las denuncias y casos que reciben en sus dependencias, ya a la realización de talleres de sensibilización sobre bullying y bullying homofóbico y por motivos raciales.

También existen iniciativas importantes desde el Poder Judicial, como la formación en temas de matoneo y cibermatoneo y justicia restaurativa, dirigido a jueces y juezas a cargo de la materia de niñez y adolescencia y de familia, coordinadas por la Escuela Judicial con el apoyo de la Comisión Nacional para el Mejoramiento de la Administración de Justicia (CONAMAJ) y la Fundación PANIAMOR. Se dispone además de un instrumento denominado "Directrices para reducir la revictimización de las personas menores de edad en procedimientos penales" (CONAMAJ, 2002). Vale señalar que existen diferentes organizaciones no gubernamentales 
que desarrollan acciones de sensibilización y atención sobre violencia escolar en general y bullying en específico. Visión Mundial Costa Rica, Defensa de Niñas y Niños-Internacional (DNI Costa Rica), Fundación Acción Joven, Fundación Omar Dengo, Fundación Arias para la Paz y el Progreso Humano son algunas de ellas.

Es importante resaltar la labor de la Fundación PANIAMOR que, desde un enfoque género-sensitivo basado en los derechos de la niñez y la adolescencia, ha desarrollado diversos programas orientados a la prevención de la violencia, de los cuales interesa mencionar "Educar sin pegar (es posible y necesario)" y "Conéctate con la Paz: Suave un toque". Además el Proyecto de Ley 18230 impulsado por esta Fundación desde 2013, "Ley especial para la Protección de los derechos de la niñez y la adolescencia frente a la violencia y el delito en el ámbito de las Tecnologías de la Información y la Comunicación y Reformas al Código Penal", incluye en los Capítulos II (Delitos contra la vida y la integridad física y psíquica de las personas menores de edad, cometidos a través de TIC) y III (Delitos contra la intimidad de las personas menores de edad, cometidos a través de TIC), los artículos 8 y 13, los cuales hacen referencia al ciberacoso entre personas menores de edad y al ciberacoso de persona menor de edad.

\section{Conclusiones}

A pesar de que en las instituciones educativas costarricenses se ha evidenciado la presencia de bullying y de un abordaje fundamentalmente punitivo (de intervención más que de prevención), no existe una legislación específica para regular la atención de este tipo de casos. Además, la normativa es limitada y a veces presenta incongruencias entre sí. Ante una problemática real, la presión mediática incremental y una mayor sensibilidad frente al bullying por parte de la ciudadanía costarricense, el Estado no está cumpliendo con las expectativas de la población, constituyéndose en un limitante para el logro pleno de sus derechos (a la educación, a la salud, a la seguridad, entre otros). Si bien es cierto que las acciones violentas se enmarcan en el ámbito educativo, esto no determina la responsabilidad única en el Ministerio de Educación Pública, sino que deben integrarse el PANI, Ministerio de Salud, Ministerio de Justicia y Paz, Instituto Nacional de la Mujer (INAMU) y todas las instancias estatales pertinentes integradas en el Consejo Nacional de Niñez y Adolescencia.

Esto implica el establecimiento de mecanismos de coordinación interinstitucional que permitan una adecuada articulación, abriendo la posibilidad de "un solo horizonte nacional y que los planes y programas repercutan en la vida cotidiana de niños, niñas y adolescentes" "De lo contrario se ejecutarán como "simples" proyectos inconexos" (Movimiento Mundial por la Infancia. Capítulo América Latina y el Caribe, 2012, p. 99).

Efectivamente, la reactivación del Consejo Nacional de Niñez y Adolescencia (CNNA) se orienta a la atención de esta necesidad, pero las acciones estatales descritas no presentan un modelo definido ni claro para el abordaje del bullying. Además, dada la multiplicidad de marcos de referencia y conceptos asociados, se limitan las posibilidades de atención y diferenciación con respecto a otros tipos de violencia escolar, centrándose en la violencia física entre el subsistema de pares, manteniendo a las espectadores secundarizados (Carozzo, 2015) y a otros subsistemas (institucional, docente, familiar) relegados (Durán, 2015).

Asimismo, las respuestas son fundamentalmente reactivas, paliativas, basadas en investigaciones y manuales realizados en otros contextos 
de los que se asumen recomendaciones genéricas incidiendo en su efectividad, con poco o ningún control que permita dar seguimiento, obtener información actualizada de posibles casos en las diferentes instituciones del país ni establecer relaciones entre factores de riesgo y protección que permitan plantear inferencias válidas sobre su efectividad tanto a nivel local como nacional (evaluación del impacto).

También, aun cuando las diferentes instancias judiciales han desarrollado propuestas e instrumentos, las prácticas revictimizadoras y la poca celeridad de la justicia en la sanción de casos de matoneo o cibermatoneo, como los expuestos en páginas previas, cuestiona la efectividad de la ruta crítica de la denuncia. Así, una de las consecuencias de la difusión pública ha sido el incremento en la búsqueda de apoyo legal que ha generado a su vez votos de la Sala Constitucional y sentencias de diferentes tribunales que establecen lineamientos al respecto.

En este sentido compartimos el planteamiento de Carozzo (2015) en cuanto a que lo legal se activa ante la inercia en el centro educativo y porque los padres y madres de familia tienen necesidad de respuestas a situaciones de agresión que victimizan a sus hijos e hijas, lo cual los obliga a recurrir al sistema legal para poder detener la situación en caso extremo. De allí la importancia de revisar la normativa actual que cubre (o no) casos de bullying para realizar las recomendaciones pertinentes pues, si bien está visibilizando y atendiendo un fenómeno contemporáneo, lo legal se focaliza en lo individual más que en lo estructural, y en enfoques punitivos, centrados en la sanción, menos efectivos que los formativos.

Tenemos importantes fortalezas para estructurar y ejecutar acciones orientadas a la prevención y atención del matoneo. Una de ellas es la fuerte plataforma de trabajo desde Cultura de Paz (Ministerio de Educación y Ministerio de Justicia y Paz por ejemplo, apoyo de la Universidad para la Paz y las ONG que fortalecen esta temática en diferentes sectores del país). Sin embargo, es necesario tomar acciones específicas frente al bullying en el corto plazo con proyecciones de mediano y largo alcance, focalizándose no solo en prevención secundaria y terciaria, sino también en la primaria, que tiende a aparecer relegada. Todo esto desde una perspectiva integral, ecológica (Bronfenbrenner, 2005) que implica la coordinación de los esfuerzos de las diferentes instancias para realizar propuestas integradas, congruentes y sistemáticas que brinden una atención efectiva a las situaciones que vive el estudiantado de nuestros centros educativos.

También pueden mencionarse las redes institucionales y las plataformas de cobertura nacional que ofrecen. Un ejemplo es el PANI y su Subsistema Local de Protección que pone a funcionar Juntas de Protección a la Niñez y la Adolescencia, conformada a su vez por Consejos Participativos de Niñez y Adolescencia. Estos son espacios de diálogo, debate, discusión, gestión de política y proyectos dirigidos a la defensa y promoción de los derechos de las personas menores de edad.

Puntualmente, para encauzar las acciones nacionales e incidir en la formulación de políticas públicas adecuadas y oportunas para una respuesta efectiva del Estado, se requiere de la integración de información y acciones que permitan:

- Generación de datos e investigación

i) Elaborar una línea base referida a temas de matoneo y cibermatoneo en el país.

ii) Integrar a los diferentes actores -más allá de los pares y de la díada matón/víctima- y subsistemas involucrados. 
iii)Dar seguimiento y medir los logros de manera sistemática y periódica.

- Desarrollo de un banco de información sobre instituciones y organizaciones no gubernamentales que trabajan sobre violencia escolar y bullying; articular experiencias, generar sinergias, trabajar en conjunto (personas y organizaciones) incentivando la comparación de datos entre países. Además, propiciar alianzas público- privadas y con organizaciones no gubernamentales para el desarrollo de proyectos de alta envergadura e integrar las entidades públicas y privadas en todos los niveles de formación. Para esto, es necesario resolver aspectos conceptuales y metodológicos, además de conocer la situación nacional en sus diferentes manifestaciones y características como punto de partida para identificar y proponer estrategias de prevención, atención y mitigación del fenómeno bullying, que refuercen los lineamientos generados por el Ministerio de Educación Pública.

- Construcción y consolidación de redes desde el saber y desde el hacer que propicien espacios de retroalimentación mutua de diferentes actores para generar impacto en las políticas públicas locales, provinciales y nacionales. Intercambiar conocimientos, experiencias y prácticas. Valorar experiencias exitosas para difundirlas y considerar su replicación. Sistematizar las lecciones aprendidas en los procesos en desarrollo, enfatizando temas de cultura organizacional, gestión y disciplina, lo que implica formación y seguimiento docente, normativa institucional y monitoreo.

- Propiciar acciones en coordinación con las universidades para fortalecer la formación de los profesionales en las áreas de Edu- cación y afines para mejorar el trabajo en los temas de convivencia, violencia escolar y bullying, brindando herramientas pedagógicas, procedimentales y legales para su atención. No puede obviarse el importante rol de los medios de comunicación en tanto actores que difunden información y generan opinión. Las estrategias de sensibilización y capacitación de los profesionales en este campo pueden redundar en un mejor manejo de los casos, el fortalecimiento de programas preventivos y la presentación de modelos positivos.

En síntesis, se propone implementar procesos de investigación e intervención que atiendan las necesidades de los actores, la adecuación conceptual y metodológica, así como el seguimiento y valoración necesarias para realizar los ajustes pertinentes a las acciones estatales actuales. Esto con el fin de propiciar directrices, políticas públicas y normativa que orienten, integren y fortalezcan las acciones necesarias para prevenir, atender y mitigar el bullying en las instituciones educativas costarricenses. Todo lo anterior desde un enfoque de fortalecimiento de la Convivencia Escolar, con acciones participativas sustentadas en las plataformas institucionales que cuenten con cobertura nacional.

\section{Referencias}

Barrantes, A. (2014, 9 de agosto) Jueces reprenden a docentes por indiferencia ante ‘bullying'. Periódico La Nación. Recuperado de http://www.nacion.com/nacional/educacion/jueces-reprenden-docentes-indiferencia-bullying_0_1431856803.html

Benites, L., Carozzo, J.; Horna, V.; Palomino, L.; Salgado, C.; Uribe, C. y Zapata, L. (2012) Bullying y convivencia en la escuela. Aspectos conceptuales, aplicativos y de investigación. 


\section{María Martha Durán}

Lima, Observatorio sobre la Violencia y Convivencia en la Escuela.

Bronfenbrenner, U. (2005). The bioecological theory of human development. En U. Bronfenbrenner (Ed.), Making human beings human: Bioecological perspectives on human development (pp. 3-15). Thousand Oaks, CA: Sage.

Cabezas, H. (2007). Detección de conductas agresivas bullyings en escolares de sexto a octavo año, en una muestra costarricense. Revista Educación de la Universidad de Costa Rica, 31(1), 123-133.

Cabezas, H. y Monge, I. (2007). ¿Qué ocurre en el aula costarricense? Los niños y las niñas que maltratan a sus compañeros. Revista Educación de la Universidad de Costa Rica, 10 (3), 1- 21.

Cabezas, H., y Monge, M. (2013). Violencia Escolar, un problema que aumenta en la escuela primaria costarricense. Revista Electrónica Actualidades Investigativas en Educación, 13 (2), pp $1-20$.

Carozzo, J. (agosto 2015) Comunicación personal. Observatorio sobre la Violencia y Convivencia en la Escuela. Lima, Perú.

Chaux, E. (2012). Educación, convivencia y agresión escolar. Colombia: Editorial Taurus.

Durán, M. (2014). Bullying y cyberbullying: desafíos de la convivencia escolar en el contexto actual. Implicaciones para la Psicología. Ponencia presentada en $V$ Congreso Regional de la Sociedad Interamericana de Psicología. San Salvador: UCA

Durán, M. (2015). Agresores, víctimas y testigos en interacción. Desarrollo de un modelo comprensivo de las conductas bullying de estudian- tes de octavo año en dos colegios de la provincia de Cartago en Costa Rica, para la formulación de una propuesta de protocolo de prevención, detección y atención. (Tesis doctoral). Universidad Estatal a Distancia, Costa Rica.

Durán, M. y Vargas, E. (2015). Análisis de caso. Implicaciones de la Sentencia Judicial 59-2014-IV contra el Estado, para el abordaje del bullying en Costa Rica. Ponencia presentada en III Congreso Internacional de Investigación Educativa 2015. San José: Universidad de Costa Rica.

Fuchs, M. (2009). Violence at german schools. Findings and open questions. International Journal of Violence and School, 9, 79-101.

Fundación Arias para la Paz y el Progreso Humano (2008). Manual 2. Programa Escuela segura, Comunidad Segura. Serie Escuela Segura-Comunidad Segura: manuales de capacitación. San José: Fundación Arias para la Paz y el Progreso Humano.

Grillo, M., y Esquivel, W. (2011). Adolescencia y TIC en Costa Rica: nuevas oportunidades, nuevos desafíos. [Versión no diagramada] En Ciberseguridad en Costa Rica (pp 82-99). Programa de la Sociedad de la Información y el Conocimiento (PROSIC): Universidad de Costa Rica.

Lentini, V.; García, C. y Borbón, J. (2013) Violencia en los colegios: características, factores explicativos y efectos. En IV Informe Estado de la Educación. San José: Programa Estado de la Nación.

Magendzo, A. y Toledo, M. (2007). Intimidación (bullying) en la escuela: investigaciones sobre clima y rendimiento escolar. Ponencia presentada en II Jornadas de coo- 
peración con Iberoamérica sobre Educación en cultura de paz. Bolivia.

Ministerio de Educación Pública (2011). Programa Convivir. Recuperado de http:// www.mep.go.cr/programas-y-proyectos/ programa-convivir

Ministerio de Educación Pública, Contraloría de Derechos Estudiantiles (2014, 2015). Estadísticas de denuncias presentadas. San José.

Movimiento Mundial por la Infancia (2012). Estado de situación de los países de Centroamérica, México, Cuba, y República Dominicana en relación con la violencia contra los niños, niñas y adolescentes en seguimiento al Estudio de Naciones Unidas sobre la Violencia contra los Niños. [Capítulo América Latina y el Caribe] San José: Visión Mundial Internacional.

Organización Mundial para la Salud (OMS) (2004). The Economic Dimensions of Interpersonal Violence. Washington: OMS. Recuperado de http://whqlibdoc.who.int/publications/2004/9241591609.pdf

Ortiz, M; Solis, K., y Umanzor, D. (2004). ¿Qué hacer ante situaciones de violencia y abuso contra personas menores de edad?: manual de procedimientos para la detección y denuncia desde el centro educativo.

Piñuel y Zabala, I. y Oñate, M. (2007). Mobbing escolar: Violencia y acoso psicológico contra los niños. Barcelona: Ed CEAC.

Programa Estado de la Nación (2011). Tercer Informe Estado de la Educación. San José. Recuperado de http://estadonacion.or.cr/ informe-iii-estado-educacion

Programa Estado de la Nación (2013). Cuarto Informe Estado de la Educación. San José.
Recuperado de http://estadonacion.or.cr/ informe-iv-estado-educacion

Programa de las Naciones Unidas para el Desarrollo - Costa Rica (PNUD-Costa Rica) (2013) Informe Nacional sobre Desarrollo Humano 2013 Aprendiendo a vivir juntos: Convivencia y desarrollo humano en Costa Rica. San José, Costa Rica.

Roland, E. (2010) Origen y primeros estudios del bullying escolar. En Ortega, R. (coord.). Agresividad injustificada, bullying y violencia escolar. Madrid: Alianza Editorial.

Ross, A. (2013, 16 de agosto) Sala IV cuida anonimato de testigos de matonismo. En Periódico La Nación. Recuperado de http:// www.nacion.com/nacional/Sala-IV-anonimato-testigos-matonismo_0_1360264019. html

Salmivalli, C.; Lagerspetz, K.; Björkqvist, K.; Österman, K.; y Kaukiainen, A. (1996). Bullying as a Group Process: Participant Roles and Their Relations to Social Status within the Group. Aggressive Behavior, 22, 1-15.

Sánchez, V. y Ortega, R. (2010). El estudio científico del fenómeno bullying. En Ortega, R. (coord.). Agresividad injustificada, bullying y violencia escolar. Madrid: Alianza Editorial.

Solis, R. (2013, 21 de marzo). Entrevista sobre bullying. Canal 9. Recuperado de http:// www.canal9.cr/hoy/2013/03/21/entrevista-sobre-bullying/

Solis, R. (2013, julio). Comunicación Personal. Ministerio de Educación Pública.

Taki, M; Slee, P.; Hymel, S.; Pepler, D.; Sim, H., y Swearer, S. (2008). A new definition and 


\section{María Martha Durán}

scales for indirect aggression en schools: results from the longitudinal comparative survey among five countries. International Journal of Violence and School, 7, 3-19.

\section{Normativa}

Comisión Nacional para el Mejoramiento de la Administración de Justicia (CONAMAJ) (2002). Directrices para reducir la revictimización de las personas menores de edad en procedimientos penales. Recuperado de http://www.conamaj. go.cr/images/libros/pdf/007a.pdf

Constitución Política de Costa Rica (1949)

Convención Internacional sobre los Derechos del Niño (1989)

Fundación PANIAMOR (2013). Proyecto de Ley 18230, Ley especial para la Protección de los derechos de la niñez y la adolescencia frente a la violencia y el delito en el ámbito de las Tecnologías de la Información y la Comunicación y Reformas al Código Penal. San José

Ley 7739. Código de la Niñez y Adolescencia (11 de diciembre 1997).

Ley Fundamental de Educación (1957)

Ley General de la Persona Joven (2002)

Ley contra el Hostigamiento Sexual en el Empleo y la Docencia $(1995,2010)$

Ley de Justicia Penal Juvenil (1996)

Ley sobre Resolución Alternativa de Conflictos (1997)

Ministerio de Educación Pública (2009). Reglamento de Evaluación de los Aprendizajes. San José.

Ministerio de Educación Pública (2009) Circular DM-005-07-2010 sobre las Acciones Institucionales para la Convivencia en los Centros Educativos. San José
Ministerio de Educación Pública (2012). Reglamento en el sector educativo sobre Ley contra el Hostigamiento Sexual en el Empleo y la Docencia .

Ministerio de Educación Pública (2012) Protocolo específico para el acoso, matonismo o bullying y el ciberbullying en los centros educativos de secundaria. San José.

Ministerio de Educación Pública (2015) Protocolo de actuación en situaciones de bullying. San José.

Ministerio de Salud (2010). Plan estratégico nacional de salud de las personas adolescentes 2010-2018. 1ㅁ․ ed. San José, Costa Rica. Recuperado de http://www.ministeriodesalud. go.cr/index.php/component/docman/doc_ view/1040-plan-estrategico-nacional-de-salud-de-las-personas-adolescentes-2010-2018

Sala Constitucional (2013). Sentencia 201310711: Recurso de amparo contra el Director del Liceo de Gravilias de Desamparados. Pronunciamiento sobre anonimato de los testigos de bullying. San José.

Sala Constitucional (2015). Sentencia 201505234: Recurso de amparo contra el Ministerio de Educación Pública, la Dirección Regional de Educación de Desamparados y la Dirección de la Escuela Finca La Capri. Pronunciamiento sobre la obligatoriedad de medidas necesarias para atender la violencia en los centros educativos. San José.

Tribunal Contencioso Administrativo y Civil de Hacienda, Sección Cuarta del Segundo Circuito Judicial de San José (2014, 11 de julio). Sentencia 59-2014: Proceso de Conocimiento interpuesto contra El Estado. San José.

Recibido: 11 de mayo de 2016 Aceptado: 30 de mayo de 2016 